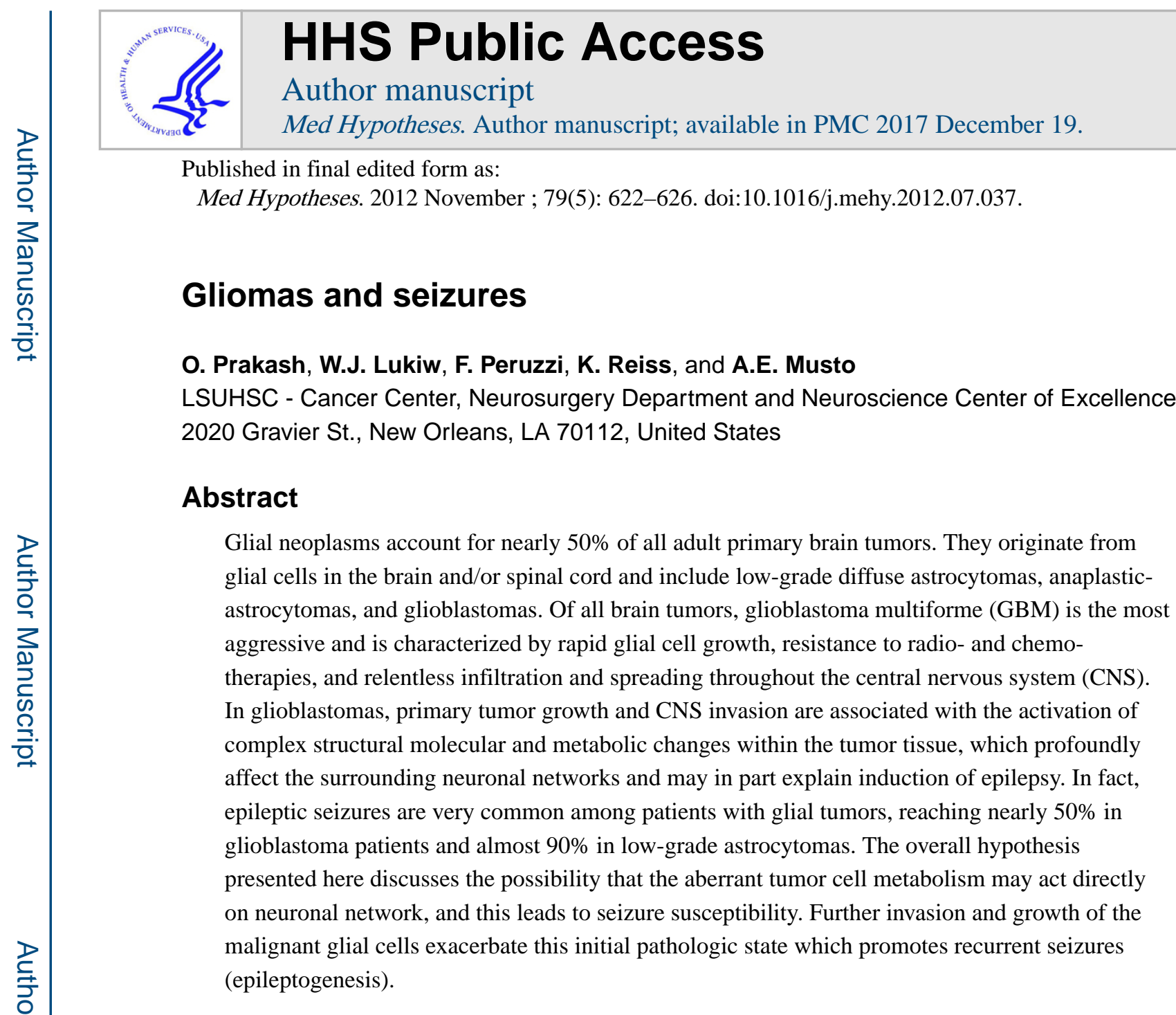

\title{
Introduction
}

Despite several advances in surgical and radiation techniques and new generation of chemotherapeutic drugs, glioblastoma multiforme (GBM) is the most invasive and undifferentiated type of brain tumor with a median survival rate of only 12 to 18 months [1]. Thus, it is essential to improve current therapies, and to limit the neurological symptoms associated with GBM such as seizures which significantly decrease quality of life [2,3]. The first step in this process is further understanding of the cellular and molecular mechanisms of GBM and underlying GBM-associate seizures so that more precisely targeted therapeutic strategies may be designed.

Seizures are common in diffuse gliomas, occurring in 50-90\% of low-grade astrocytoma patients and in 20-50\% of glioblastoma patients [4]. Seizures could be an initial symptom in $45 \%$ of patients with GBM and often represent the first symptom of this deadly disease. The partial status epilepticus is the most frequent presentation and is likely associated with

*Corresponding author. Tel.: +1 504599 0846. amusto@1suhsc.edu (A.E. Musto).

Conflict of interest statement

None declared. This work was departmentally supported. 
edema and/or neuroinflammation [2]. Although, epilepsy is more frequent in low-grade gliomas, seizures are more difficult to control in high-grade gliomas [5].

GBM associated with epilepsy is a complex neurological disorder. Although a single seizure could be the first manifestation of GBM, the seizures associated with GBM became recurrent in frequency and in severity. This process of secondary epileptogene-sis, involves several molecular and cellular rearrangements of neuronal networks. Like in primary epileptogenesis there are no drugs to abort the secondary epileptogenesis process [6]. Although, some antileptic drugs could attenuate the frequency of seizures and improve the survival of patients with GBM, a single seizure may aggravate the brain to develop status epilepticus and engender its clinical complications. Also, the antiepileptic drug toxicities substantially contribute to neurological morbidity in at least $25 \%$ of patients during followup, thus worsening the quality of life for the patient and interfering with clinical response criteria evaluation in the absence of radiologic evidence [3]. Therefore, the ultimate goal of our research is to identify molecular bases of tumor-induced seizures and to develop new clinical modalities, which in parallel to the anticancer treatment will target epileptic seizures. Here, we propose a new hypothetical mechanism of tumor-induced epilepsy, which may serve as a template for the development of early therapeutic interventions.

Hypothesis (Fig. 1): Our hypothesis states that GBM triggers molecular cascades that induce hyperexcitability in the surrounding neuronal network. The abnormal activation of Src family kinases, Lyn and Fyn, in particular, within intra- and peritumoral tissue triggers complex signaling responses which promote glutamate accumulation, increase of gap junction, and NMDA receptor activation in the peritumoral neuronal network, ultimately leading to the development of epileptic seizures.

\section{Evaluation of the hypothesis}

Several molecular pathways are proposed in the etiology of GBM $[7,8]$. But how these pathways are involved or related to epileptogenesis is largely unknown. In large number of cases the epileptic seizure is thought to arise in the peritumoral tissue and not in the tumor itself $[9,10]$. In such cases, resection of the peritumoral zone was sufficient to control the seizure, however, in other studies, tumor resection alone led to seizure control [11]. Thus, intratumoral and/or peritumoral molecular pathways activated by impaired glial cell function, disordered neuronal cell connectivity, and likely other factors could explain the induction of epileptogenesis. Here, we propose some common mechanisms relevant for GBM evolution which lead to epileptogenesis.

\section{Intratumoral pathways}

Src family kinases (SFKs) are non-receptor tyrosine kinases which mediate signaling pathways in cell proliferation, differentiation, invasion, and metastasis. Among the SFKs, Fyn and Lyn are known to show high expression in the central nervous system [12]. Our analysis using the Rembrandt data base revealed a twofold upregulation of Lyn and 1.5-fold upregulation in Fyn in glioblastoma samples compared to the non-neoplastic brain tissue ( $P$ $<0.001)$ [13]. There was no significant change in the expression of Src. Kaplan Meier Plot 
analysis of patients with upregulation of Lyn and Fyn revealed significantly worse survival compared to rest of the gliomas $P<0.001$ ) [13]. Other studies also support the role of Lyn and Fyn kinases in the pathogenesis of GBM. Lyn kinase activity accounts for $>90 \%$ of the pan-Src activity in glioblastoma tumors and suggests its role in promoting the highly migratory/invasive phenotype in these tumors [14]. Additionally, integrins $a_{v} \beta_{3}$ and $a_{v} \beta_{5}$ are upregulated in glioblastoma tumors [15,21]. Downregulation of Lyn expression inhibits cell migration mediated by $a_{v} \beta_{3}$ integrins which are upregulated in GBM tumors [15]. In addition, Lyn is involved in the signaling of integrin and platelet-derived growth factor receptors (PDGFR) which are upregulated in GBM cells [15]. Lyn also enhances motility of epidermal growth factor receptor (EGFR)-expressing glioblastoma cells [15]. EGFR is overexpressed in approximately 50\% GBMs and its activation is linked to cell proliferation, migration, metastases and cell survival [16]. There is evidence that GBM patients whose tumors exhibit activated EGFR signaling also frequently display activated Fyn and Src which promote tumor motility and survival [16]. Fyn constitutively associates with integrin $a_{v} \beta_{5}$ [15]. Inhibition of Fyn and Src by either genetic or pharmacological interventions greatly reduces tumor invasion and promotes tumor cell apoptosis [16].

High expression of Lyn and Fyn kinases in the central nervous system [12,17], may contribute to GBM -associated epileptogenesis. Fyn mediated signaling is essential for normal development of the central nervous system and brain functions [18,19]. Earlier studies limited Fyn expression to neurons and oligodendrocytes, but now there is evidence that Fyn is highly expressed in the astrocytes involved in reactive gliosis [19]. Also, Fyn plays an important role in synaptic plasticity [20], suggesting that it may be involved in epileptogenesis, and could impair the regulation of neuron-astrocyte communication. Transgenic mice expressing constitutively active Fyn exhibited enhanced excitatory functions, higher incidence of spontaneous seizures, and higher mortality [21]. On the other hand, no obvious epileptic activity was noticed in mice overexpressing native Fyn. The study also showed that Fyn phosphorylated NMDA receptor subunits NR2A and NR2B, preferentially NR2B. Using kindling and pilocarpine rat models of limbic epilepsy, another study found that activation of NR2A- but not NR2B-containing NMDARs was required for epileptogenesis, but both types were involved in seizure-induced neuronal cell death [22]. In addition to Fyn, Src phosphorylates NR2A subunit to enhance NMDA receptor activity [23]. Thus, suggesting a strong positive relationship between activation of NMDA receptor NR2A and the development of epileptic seizures.

Focal adhesion kinase (FAK) is a non-receptor tyrosine kinase which is upregulated in glioblastoma cells [24]. The activated FAK then binds to Src family kinases and other signaling molecules to activate pro-migratory and proliferative signaling pathways that contribute to cell survival, proliferation, and migration, etc. FAK promotes glioblastoma cell proliferation in an intracerebral xenograft model [24]. In another study, specific knockdown of FAK expression in glioblastoma cells increased survival compared to control mice [25]. Similar results were also seen when expression of another focal adhesion kinase Pyk2 was knocked down in GBM cells [25]. Because GBM disease progression involves a complex interplay between tumor cells and peritumoral microenvironment, multiple signaling pathways activated by focal adhesion kinases might contribute to tumor-associated epileptogenesis. 
Glutamine synthetase (GS) deficiency in GBM cells may be coupled with epileptogenesis [5]. Lower levels of GS in brain tumor samples are observed in epileptogenic GBMs as compared with non-epileptogenic GBMs, suggesting a down-regulation of GS may have an important pro-epileptogenic role in GBM [5]. Decreased GS results in accumulation of glutamate by tumor cells in the peritumoral fluid [5]. The measured glutamate (Glu) release from gliomas is large enough to suggest an over-activation of neuronal Glu receptors that activates neuronal network leading to clinical seizures [26]. Also glutamate plays a vital role in the proliferation and invasion of GBM tumors by autocrine or paracrine loops through $\mathrm{Ca}^{2+}$-permeable AMPA glutamate receptors [9,27]. AMPA receptors are highly expressed in glioblastoma and perform critical functions in glioma biology and enhance its malignant phenotype. AMPA receptors on glioma cells may regulate cell motility and tumor invasiveness. AMPA receptors can also function as cell-surface signal transducers through their association with Lyn kinase [12]. Activation of Lyn through AMPA receptors is independent of $\mathrm{Ca}^{2+}$ leading to activation of MAPK signaling pathway and the expression of brain-derived neurotrophic factor (BDNF) which may contribute to epileptic seizures $[12,28]$. In another study, Lyn kinase activity was found to be almost 20 -fold higher in the mast cells from epilepsy-prone EL mice versus epilepsy-resistant ASK mice, suggesting a role of Lyn kinase in the pathogenic process of epileptogenesis [29]. Further, there is evidence that glutamate increases the expression of EGFR on GBM cells and trigger cell proliferation via EGFR-phospho-Akt pathway [30].

Eph receptor tyrosine kinases represent the largest family of receptor tyrosine kinases. EphA4 expression is increased in high grade gliomas compared to normal brain tissue [31]. Further, EphA4 formed complex with FGFR1 in the cells and potentiated cell proliferation and migration through accelerating the FGFR signaling pathway [31]. Ephrin ligands have been suggested to regulate neuron-glia interactions. Ephrin A3, ligand of EphA4, overexpression in astrocytes increases susceptibility to excitotoxicity and seizures, and reduces glutamate transporters. Lack of EphA4 or ephrin-A3 increases the abundance of glutamate transporters which results in more efficient removal of glutamate [32]. On the other hand, reduction of glial glutamate transporters increases synaptic glutamate concentration causing excitotoxicity which may lead to epilepsy and neurodegeneration [33]. These results suggest that EphA4-mediated signaling plays a role in the aggressiveness of GBM, and also provides a critical mechanism for astrocytes to regulate synaptic function and plasticity [32].

\section{Peritumoral pathways}

Propagation and amplification of cell injury during tumor growth and invasion may also play a role in the generation and propagation of seizure activity [34,35]. Epileptiform activity is developed in the peripheral glioma invasion area, where single neurons are surrounded by a very few tumor cells [36]. EEG studies of glioma patients revealed that epileptiform activity was usually localized at the tumor "border" [37]. Somatostatin was reduced in these regions, suggesting loss of inhibitory mechanisms and over-expression of the NMDA receptor $[22,38,39]$. Furthermore, the gap junction protein connexin-43 (CX43) is increased in peritumoral astrocytes, suggesting reorganization of the astrocytic syncytium, possibly contributing to tumor-related seizures [40]. On the other hand, increased gap-junction 
expression or coupling has been implicated in the synchronization of the discharge at the epileptic focus that contributes seizure onset, maintenance, and propagation of the epileptic focus [41].

Glutamate released from gliomas is large enough to overactivate neuronal glutamate receptors and mediate neuroexcitotoxicity [42] which may eventually lead to epilepsy and neurodegeneration $[33,43]$. Further, the extracelluar accumulation of glutamate could target aberrant activation of neuronal NMDA receptors in the peritumoral tissue manifesting as hyperexcitability and may explain the frequent occurrence of seizures emanating from the tumor and surrounding brain.

Micro RNAs (miRNAs) are a family of short, endogenous non-coding RNAs that are posttranscriptional regulators of gene expression. Although they are extensively expressed in the CNS in physiological conditions, their role in neurological disorders remains largely unknown. Nevertheless, increasing evidence suggests that miRNAs are dysregulated in a surprisingly wide range of human diseases including GBM [44,45]. Indeed specific brainenriched miRNAs such as miRNA-125b have been shown to be responsible for the regulation of glial cell proliferation, and cell cycle regulatory molecules such as the cyclindependent kinase inhibitor $2 \mathrm{~A}(\mathrm{CDKN} 2 \mathrm{~A})$ that are responsible for the regulation of glial cell proliferation in brain cells $[44,46]$.

In addition to cellular miRNAs, increasing evidence show that glioblastoma cells release microvesicles (MVs), which contain a subset of proteins, DNA, mRNAs and non-coding RNAs, including miRNAs. MVs are 30-500 $\mathrm{nm}$ in diameter and they are shed by most of the cells in normal and pathological conditions [47]. Glioblastomas release the highest amount of MVs. Tumor-derived MVs have been shown to "instruct" normal cells in the tumor microenvironment in favor of tumor growth, invasion, and immune suppression [48]. Both, mRNAs and miRNAs transferred from cell to cell by MVs, can be functional and affect gene expression in the recipient cells $[48,49]$. There are no reports on the role of GBM-derived MVs and epilepsy; however, we could reasonably speculate that inflammation might be a common denominator between tumors and epilepsy. The immunological content of membrane-enclosed MVs (called exosomes) has been shown to be immunogenic and capable of antitumor, as well as immunosuppressive activities [50]. miR-146a/b, a negative regulator of the immune response, belongs to the miRNA signature of ten miRNAs that predict survival in glioblastoma patients [51]. Prominent upregulation of miR-146a has been observed in the hippocampus of a rat model of temporal lobe epilepsy [52], and could be a source for inflammation that induces hyperexcitability related with seizures [53]. Another example is provided by miR-221 and miR-21, which are highly expressed in glioblastomas and other malignancies [45,54,55]. Inhibition of miR-21 has been shown to induce apoptosis, reduce invasiveness, and to suppress tumorigenic activity [56]. Of interest, miR-21 is up-regulated in rat hippocampus following lithium-pilocarpine-induced status epilepticus [57]. This up regulation could mediate regulation of connexin-43 in glialneuronal communication and glial proliferation especially because this connexin is expressed in interneurons which are vulnerable in epilepsy [58]. As previously mentioned, no empirical connection has been established between miRNAs, GBM-derived MVs, and epilepsy. Taken together, it is very likely that the miRNA-mediated dysregulated cellular 
pathways may modify essential components of synaptic plasticity in the tumoral and peritumoral tissues and modulate the development of epileptogenesis process.

\section{Concluding remarks}

The ultimate goal of the translational research is to identify molecular bases of tumorinduced seizures and to develop new clinical modalities, which in parallel to the anticancer treatment will target epileptic seizures. Several mechanisms hypothesized in this article are possibly involved (Fig. 1). The identification of specifically tumoral and peritumoral new molecular clues to the mechanisms of glial cell proliferation, should provide novel pharmacological targets for the clinical management of brain cancers and the associated epileptogenesis.

\section{Acknowledgments}

The authors thank Drs. Nicolas G. Bazan and Frank Culicchia for their support, and Darlene Guillot, Kenia Carvajal and Carter Guise for their technical assistance.

\section{References}

1. Adamson C, Kanu OO, Mehta AI, et al. Glioblastoma multiforme: a review of where we have been and where we are going. Expert Opin Investig Drugs. 2009; 18:1061-83.

2. Moots PL, Maciunas RJ, Eisert DR, Parker RA, Laporte K, Abou-Khalil B. The course of seizure disorders in patients with malignant gliomas. Arch Neurol. 1995; 52:717-24. [PubMed: 7619029]

3. Riva M, Salmaggi A, Marchioni E, et al. Tumour-associated epilepsy: clinical impact and the role of referring centres in a cohort of glioblastoma patients. A multicentre study from the Lombardia Neurooncology Group. Neurol Sci. 2006; 27:345-51. [PubMed: 17122945]

4. Ruda R, Trevisan E, Soffietti R. Epilepsy and brain tumors. Curr Opin Oncol. 2010; 22:611-20. [PubMed: 20706121]

5. Rosati A, Marconi S, Pollo B, et al. Epilepsy in glioblastoma multiforme: correlation with glutamine synthetase levels. J Neurooncol. 2009; 93:319-24. [PubMed: 19183851]

6. Pitkanen A, Lukasiuk K. Mechanisms of epileptogenesis and potential treatment targets. Lancet Neurol. 2011; 10:173-86. [PubMed: 21256455]

7. Kraksta C, Chekenya M. Survival signalling and apoptosis resistance in glioblastomas: opportunities for targeted therapeutics. Mol Cancer. 2010; 9:135. [PubMed: 20515495]

8. Akhavan D, Cloughesy TF, Mischel PS. MTOR signaling in glioblastoma: lessons learned from bench to bedside. Neuro Oncol. 2010; 12:882-9. [PubMed: 20472883]

9. de Groot J, Sontheimer H. Glutamate and the biology of gliomas. Glia. 2010; 59:1181-9. [PubMed: 21192095]

10. de Groot M, Toering ST, Boer K, et al. Expression of synaptic vesicle protein 2A in epilepsyassociated brain tumors and in the peritumoral cortex. Neuro Oncol. 2010; 12:265-73. [PubMed: 20167814]

11. Mikuni N, Ikeda A, Takahashi JA, et al. A step-by-step resection guided by electrocorticography for nonmalignant brain tumors associated with long-term intractable epilepsy. Epilepsy Behav. 2006; 8:560-4. [PubMed: 16495156]

12. Hayashi T, Umemori H, Mishina M, Yamamoto T. The AMPA receptor interacts with and signals through the protein tyrosine kinase Lyn. Nature. 1999; 397:72-6. [PubMed: 9892356]

13. Velcheti, V., Levitt, D., Prakash, O. Preclinical evaluation of bafetinib for treatment of glioblastoma multiforme. American Society of Clinical Oncology, Annual Meeting; 2010.

14. Stettner MR, Wang W, Nabors LB, et al. Lyn kinase activity is the predominant cellular SRC kinase activity in glioblastoma tumor cells. Cancer Res. 2005; 65:5535-43. [PubMed: 15994925] 
15. Ding Q, Stewart J Jr, Olman MA, Klobe MR, Gladson CL. The pattern of enhancement of Src kinase activity on platelet-derived growth factor stimulation of glioblastoma cells is affected by the integrin engaged. J Biol Chem. 2003; 278:39882-91. [PubMed: 12881526]

16. Lu KV, Zhu S, Cvrljevic A, et al. Fyn and SRC are effectors of oncogenic epidermal growth factor receptor signaling in glioblastoma patients. Cancer Res. 2009; 69:6889-98. [PubMed: 19690143]

17. Umemori H, Wanaka A, Kato H, Takeuchi M, Tohyama M, Yamamoto T. Specific expressions of Fyn and Lyn, lymphocyte antigen receptor-associated tyrosine kinases, in the central nervous system. Brain Res Mol Brain Res. 1992; 16:303-10. [PubMed: 1337939]

18. Narisawa-Saito M, Silva AJ, Yamaguchi T, Hayashi T, Yamamoto T, Nawa H. Growth factormediated Fyn signaling regulates alpha-amino-3- hydroxy-5-methyl-4-isoxazolepropionic acid (AMPA) receptor expression in rodent neocortical neurons. Proc Natl Acad Sci U S A. 1999; 96:2461-6. [PubMed: 10051665]

19. Chun JT, Crispino M, Tocco G. The dual response of protein kinase Fyn to neural trauma: early induction in neurons and delayed induction in reactive astrocytes. Exp Neurol. 2004; 185:109-19. [PubMed: 14697322]

20. Bjarnadottir M, Misner DL, Haverfield-Gross S, et al. Neuregulin1 (NRG1) signaling through Fyn modulates NMDA receptor phosphorylation: differential synaptic function in NRG1+/- knockouts compared with wild-type mice. J Neurosci. 2007; 27:4519-29. [PubMed: 17460065]

21. Kojima N, Ishibashi H, Obata K, Kandel ER. Higher seizure susceptibility and enhanced tyrosine phosphorylation of N-methyl-D-aspartate receptor subunit 2B in fyn transgenic mice. Learn Mem. 1998; 5:429-45. [PubMed: 10489260]

22. Chen JW, Naylor DE, Wasterlain CG. Advances in the pathophysiology of status epilepticus. Acta Neurol Scand Suppl. 2007; 186:7-15. [PubMed: 17784531]

23. Huo JZ, Dykstra CM, Gurd JW. Increase in tyrosine phosphorylation of the NMDA receptor following the induction of status epilepticus. Neurosci Lett. 2006; 401:266-70. [PubMed: 16600505]

24. Natarajan M, Hecker TP, Gladson CL. FAK signaling in anaplastic astrocytoma and glioblastoma tumors. Cancer J. 2003; 9:126-33. [PubMed: 12784878]

25. Lipinski CA, Tran NL, Viso C, et al. Extended survival of Pyk2 or FAK deficient orthotopic glioma xenografts. J Neurooncol. 2008; 90:181-9. [PubMed: 18648907]

26. Morimoto K, Fahnestock M, Racine RJ. Kindling and status epilepticus models of epilepsy: rewiring the brain. Prog Neurobiol. 2004; 73:1-60. [PubMed: 15193778]

27. Ishiuchi S. New roles of glutamate receptors in glias and gliomas. Brain Nerve. 2009; 61:753-64. [PubMed: 19618852]

28. Scharfman HE. Brain-derived neurotrophic factor and epilepsy-a missing link? Epilepsy Curr. 2005; 5:83-8. [PubMed: 16145610]

29. Kitaura J, Kawakami Y, Maeda-Yamamoto M, Horejsi V, Kawakami T. Dysregulation of Src family kinases in mast cells from epilepsy-resistant ASK versus epilepsy-prone EL mice. J Immunol. 2007; 178:455-62. [PubMed: 17182584]

30. Schunemann DP, Grivicich I, Regner A, et al. Glutamate promotes cell growth by EGFR signaling on U-87MG human glioblastoma cell line. Pathol Oncol Res. 2010; 16:285-93. [PubMed: 19997873]

31. Fukai J, Yokote H, Yamanaka R, Arao T, Nishio K, Itakura T. EphA4 promotes cell proliferation and migration through a novel EphA4-FGFR1 signaling pathway in the human glioma U251 cell line. Mol Cancer Ther. 2008; 7:2768-78. [PubMed: 18790757]

32. Filosa A, Paixao S, Honsek SD, et al. Neuron-glia communication via EphA4/ephrin-A3 modulates LTP through glial glutamate transport. Nat Neurosci. 2009; 12:1285-92. [PubMed: 19734893]

33. Tanaka K, Watase K, Manabe T, et al. Epilepsy and exacerbation of brain injury in mice lacking the glutamate transporter GLT-1. Science. 1997; 276:1699-702. [PubMed: 9180080]

34. Henshall DC, Murphy BM. Modulators of neuronal cell death in epilepsy. Curr Opin Pharmacol. 2008; 8:75-81. [PubMed: 17827063]

35. Kohling R, Senner V, Paulus W, Speckmann EJ. Epileptiform activity preferentially arises outside tumor invasion zone in glioma xenotransplants. Neurobiol Dis. 2006; 22:64-75. [PubMed:

16309916] 
36. Senner V, Kohling R, Puttmann-Cyrus S, Straub H, Paulus W, Speckmann EJ. A new neurophysiological/neuropathological ex vivo model localizes the origin of glioma-associated epileptogenesis in the invasion area. Acta Neuropathol. 2004; 107:1-7. [PubMed: 13680280]

37. Patt S, Steenbeck J, Hochstetter A, et al. Source localization and possible causes of interictal epileptic activity in tumor-associated epilepsy. Neurobiol Dis. 2000; 7:260-9. [PubMed: 10964598]

38. Vezzani A, Hoyer D. Brain somatostatin: a candidate inhibitory role in seizures and epileptogenesis. Eur J Neurosci. 1999; 11:3767-76. [PubMed: 10583466]

39. Galanopoulou AS, Moshe SL. The epileptic hypothesis: developmentally related arguments based on animal models. Epilepsia. 2009; 50(Suppl 7):37-42.

40. Aronica E, Gorter JA, Jansen GH, Leenstra S, Yankaya B, Troost D. Expression of connexin 43 and connexin 32 gap-junction proteins in epilepsy-associated brain tumors and in the perilesional epileptic cortex. Acta Neuropathol. 2001; 101:449-59. [PubMed: 11484816]

41. LeBeau FE, Traub RD, Monyer H, Whittington MA, Buhl EH. The role of electrical signaling via gap junctions in the generation of fast network oscillations. Brain Res Bull. 2003; 62:3-13. [PubMed: 14596887]

42. Takano T, Lin JH, Arcuino G, Gao Q, Yang J, Nedergaard M. Glutamate release promotes growth of malignant gliomas. Nat Med. 2001; 7:1010-5. [PubMed: 11533703]

43. Chung WJ, Lyons SA, Nelson GM, et al. Inhibition of cystine uptake disrupts the growth of primary brain tumors. J Neurosci. 2005; 25:7101-10. [PubMed: 16079392]

44. Pogue AI, Cui JG, Li YY, Zhao Y, Culicchia F, Lukiw WJ. Micro RNA-125b (miRNA-125b) function in astrogliosis and glial cell proliferation. Neurosci Lett. 2010; 476:18-22. [PubMed: 20347935]

45. Lukiw WJ, Cui JG, Li YY, Culicchia F. Up-regulation of micro-RNA-221 (miRNA-221; chr Xp11. 3 ) and caspase-3 accompanies down-regulation of the survivin-1 homolog BIRC1 (NAIP) in glioblastoma multiforme (GBM). J Neurooncol. 2009; 91:27-32. [PubMed: 18759060]

46. Sethi P, Lukiw WJ. Micro-RNA abundance and stability in human brain: specific alterations in Alzheimer's disease temporal lobe neocortex. Neurosci Lett. 2009; 459:100-4. [PubMed: 19406203]

47. Thery C, Zitvogel L, Amigorena S. Exosomes: composition, biogenesis and function. Nature Rev. 2002; 2:569-79.

48. Skog J, Wurdinger T, van Rijn S, et al. Glioblastoma microvesicles transport RNA and proteins that promote tumour growth and provide diagnostic biomarkers. Nat Cell Biol. 2008; 10:1470-6. [PubMed: 19011622]

49. Zhang Y, Liu D, Chen X, et al. Secreted monocytic miR-150 enhances targeted endothelial cell migration. Mol cell. 2010; 39:133-44. [PubMed: 20603081]

50. Valenti R, Huber V, Iero M, Filipazzi P, Parmiani G, Rivoltini L. Tumor-released microvesicles as vehicles of immunosuppression. Cancer Res. 2007; 67:2912-5. [PubMed: 17409393]

51. Srinivasan S, Patric IR, Somasundaram K. A ten-microRNA expression signature predicts survival in glioblastoma. PLoS One. 2011; 6:e17438. [PubMed: 21483847]

52. Aronica E, Fluiter K, Iyer A, et al. Expression pattern of miR-146a, an inflammation-associated microRNA, in experimental and human temporal lobe epilepsy. Eur J Neurosci. 2010; 31:1100-7. [PubMed: 20214679]

53. Musto AE, Samii M. Platelet-activating factor receptor antagonism targets neuroinflammation in experimental epilepsy. Epilepsia. 2011; 52:551-61. [PubMed: 21204830]

54. Zhou X, Ren Y, Moore L, et al. Downregulation of miR-21 inhibits EGFR pathway and suppresses the growth of human glioblastoma cells independent of PTEN status. Lab Invest. 2010; 90:144-55. [PubMed: 20048743]

55. Rao X, Di Leva G, Li M, et al. MicroRNA-221/222 confers breast cancer fulvestrant resistance by regulating multiple signaling pathways. Oncogene. 2011; 30:1082-97. [PubMed: 21057537]

56. Papagiannakopoulos T, Shapiro A, Kosik KS. MicroRNA-21 targets a network of key tumorsuppressive pathways in glioblastoma cells. Cancer Res. 2008; 68:8164-72. [PubMed: 18829576] 
57. Hu K, Zhang C, Long L, et al. Expression profile of microRNAs in rat hippocampus following lithium-pilocarpine-induced status epilepticus. Neurosci Lett. 2011; 488:252-7. [PubMed: 21094214]

58. Elias LA, Turmaine M, Parnavelas JG, Kriegstein AR. Connexin 43 mediates the tangential to radial migratory switch in ventrally derived cortical interneurons. J Neurosci. 2010; 30:7072-7. [PubMed: 20484649] 


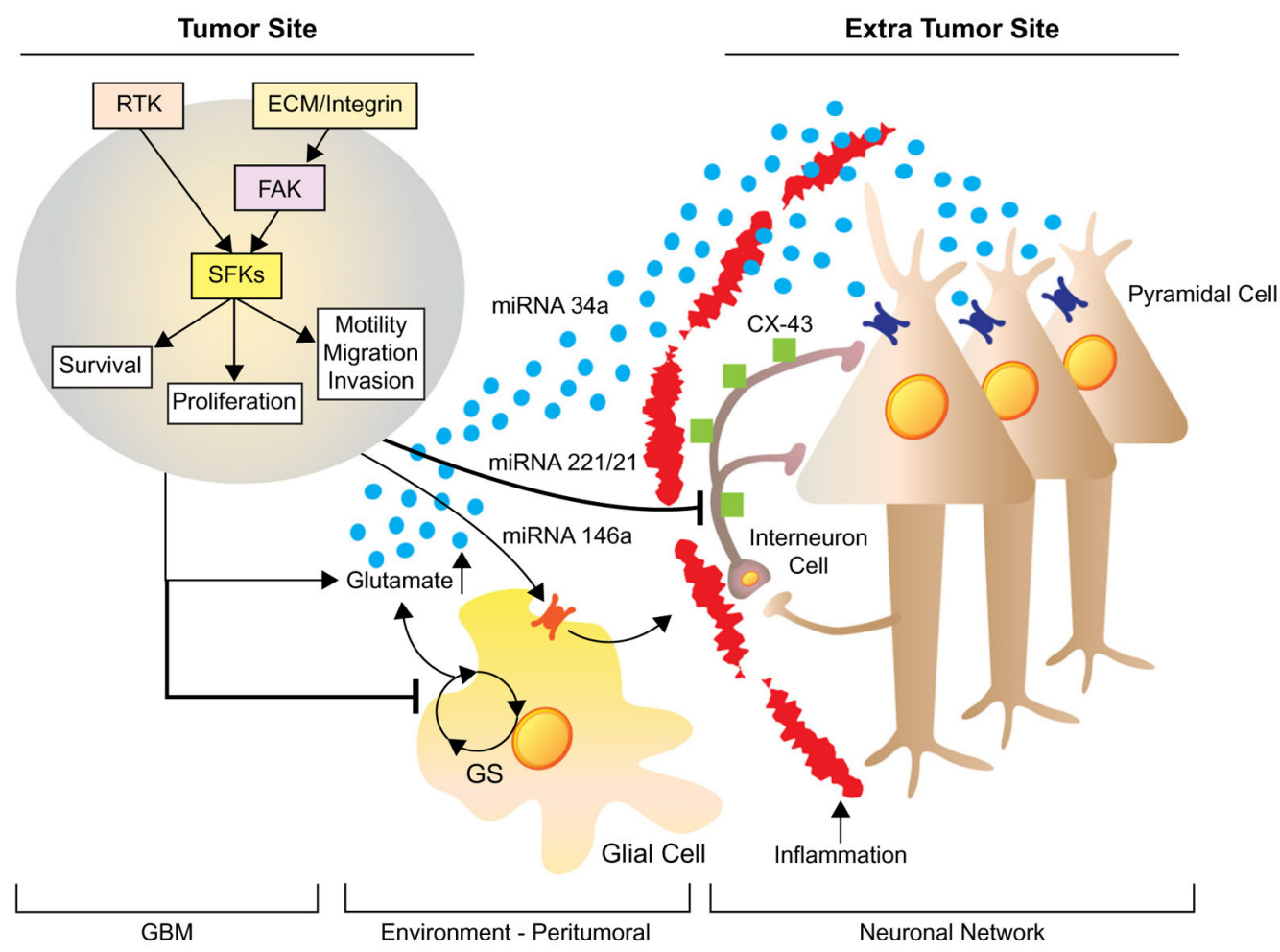

Fig. 1.

Hypothesis for the influence of GBM-produced factors on epileptogenesis. Tumor expression of receptor tyrosine kinases and extracellular matrix protein integrins activates focal adhesion kinase and Src family kinases directly and indirectly, respectively. SKF's activation promotes survival, proliferation and invasion. These molecular tumoregenic processes lead to accumulation of glutamate and miRNAs in the peritumoral area. Glutamate accumulation is exacerbated by malfunctioning of glial cells in the peritumoral area due to downregulation of glutamate synthetase. In addition, miRNAs and glutamate induce increase of connexins in the neighboring interneuronal network. The aberrant expression of connexins, N-methyl-d-aspartate and miRNAs in principal neurons strengthens the neuronal circuit synchronization of abnormal neuronal activity leading to seizures. 